\title{
Grandir au contact de la résistance
}

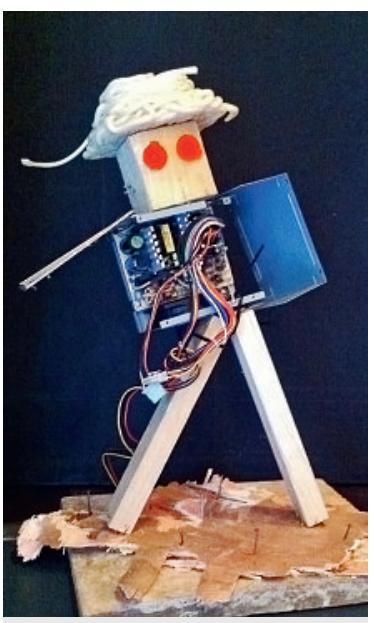

Grandi au contact de la résistance? Travail d'une collaboratrice à l'hôpital d'Affoltern am Albis.

On ne les connaît que trop, ces slogans du genre «la crise est une chance» ou «grandir au contact de la résistance». Aussi usés qu'ils puissent être, ils contiennent une part de vérité. L'image de l'athlète désireux d'obtenir un paquet de muscles impressionnants à l'aide d'appareils de musculature peut être reportée cum grano salis sur des situations historiques, sociales et interrelationnelles.

Chaque maladie étant à comprendre comme une crise, les médecins devraient être en mesure de gérer les crises. Mais quelle est la situation lorsque le système de santé est lui-même malade, entre en crise, comme on l'a vu ces dernières années dans les discours officiels? Qui a les meilleures recettes en stock?

Indépendamment du fait que les recettes convaincantes sont généralement lacunaires, le discours officiel en matière de santé est actuellement dominé par les économistes et les politiciens dans bien des domaines. Pas étonnant dès lors de voir généralement les débats tourner d'abord autour des questions financières puisque la performance et la qualité de notre système de santé ne sont guère contestées.

Rationalisation, augmentation de l'efficacité, contrôles et transparence de la qualité et concurrence en tant que remède miracle sont les maîtres mots sensés diriger notre système de santé. Jusqu'à ce jour, il en résulte pour le corps médical (comme d'ailleurs pour d'autres groupes professionnels tels les enseignants) avant tout une bureaucratie galopante et une diminution du temps à disposition pour les tâches «essentielles», c'est-à-dire pour les patients.

\section{Chaque maladie étant à comprendre comme une crise, les médecins devraient être en mesure de gérer les crises}

* Vous trouverez de plus amples informations sur www.paulvogt.com

Aussi la question se pose de savoir si le chemin choisi va dans la bonne direction. Ou si nous devons simplement le suivre encore un moment, parvenir à son extrémité pour découvrir plus clairement que nous sommes parvenus à un cul-de-sac et que nous devons nous réorienter d'urgence et viser autre chose?

Quoi qu'il en soit: le corps médical endosse un rôle décisif dans ce processus. Il voit de près les répercussions de l'évolution actuelle sur les patients et c'est son devoir de s'engager. Faut-il choisir la voie de la collaboration en exerçant une critique constructive, à l'instar de la FMH qui a opté pour les forfaits DRG et les soins intégrés, ou faut-il se mettre dans l'opposition, à l'instar de certains groupements du corps médical qui ne doivent pas être négligés?
Comme personne ne sait qu'elle en sera l'issue, les deux positions se justifient.

Ces deux positions engendrent des personnalités au profil aiguisé. Le symposium organisé récemment à Affoltern près de Zurich et intitulé «Wa(h)re Medizin» (jeu de mot signifiant: vraie médecine ou médecine, objet de consommation) ressemblait au village gaulois d'Astérix, dernier bastion de la résistance aux légions de Jules César car on pouvait y côtoyer quelques irréductibles. Ceux-ci n'avaient pas de potion magique à nous offrir mais des analyses percutantes.

Par exemple le professeur Paul R. Vogt. Ce spécialiste en chirurgie thoracique et cardio-vasculaire dispose d'un excellent réseau international et connaît de première main les tendances nationales, européennes et mondiales. Il a été entre autres professeur ordinaire de 2000 à 2006 pour la chirurgie cardio-vasculaire et pédocardiaque à la clinique universitaire de Giessen, en Allemagne, il a assumé la fonction de directeur médical du «Beijing Focus Century International Medical Exchange» et il a enseigné en tant que professeur invité dans différentes universités chinoises.

Ce rhétoricien à la langue acérée argumente avec esprit, manie un humour laconique, ne parle pas la langue de bois et ne ménage personne. Sujet de la discussion: les contrôles de la qualité: «Ces contrôles existent-ils en médecine?» demande-t-il, et il répond aussitôt «non». «Chacun peut-il faire ce qu'il veut? - oui». «Les données officielles sont-elles sincères? - non». «Est-ce que l'on nous ment? - massivement». «Pourquoi? - Pour des motifs d'argent, de gloire, de pouvoir». «La politique peut-elle faire quelque chose? - non».

Paul R. Vogt n'en reste pas aux slogans, mais il étaye ses thèses avec des données solides; il formule aussi des propositions concrètes et réalisables sur la manière d'augmenter la qualité en médecine. * A cet effet, il se focalise clairement sur le postulat suivant: «Ce sont les disciplines invasives et onéreuses qui doivent être contrôlées et non pas les médecins de famille qui soignent les migraines et les douleurs dorsales chroniques.» Il exige entre autres des contrôles à l'improviste, tels qu'on les réalise dans le sport de pointe.

Les esprits critiques comme Paul R. Vogt ne suscitent à coup sûr pas uniquement de l'enthousiasme, ils sont au contraire insupportables pour beaucoup. Cependant, même ses adversaires devraient lui être reconnaissants. En énonçant des questions inconfortables et des vérités désagréables, il contraint ses opposants à devenir eux-mêmes créatifs, à s'emparer des problèmes et à développer des solutions. Si notre système de santé avance, on le doit essentiellement à ce genre de forces. Chaque partie grandit au contact de la résistance qu'on lui oppose.

Bruno Kesseli 